\title{
Grain Boundary Precipitation in Ni Based Superalloy 690 Investigated via Site- specific Atom Probe Microscopy
}

\author{
Bharat Gwalani ${ }^{1}$, Talukder Alam ${ }^{1}$, Mike Kaufman ${ }^{2}$, Raj Banerjee ${ }^{1}$ \\ 1. Center for Advanced Research and Technology and Department of Materials Science and \\ Engineering, University of North Texas, Denton, TX, USA \\ 2. Department of Metallurgical and Materials Engineering, Colorado School of Mines, Golden, CO, USA
}

Alloy 690 has been proposed to be an alternative to Alloy 600 for use in tubing materials that carry the super-heated steam onto the turbine blades of land-based nuclear power generators. Alloy 690 is known to be more resistant to primary water stress corrosion cracking than alloy 600 , consequently aiding in the life extension process of nuclear reactors [1]. A thorough study of the long term aging response of alloy 690 is yet to be done in great detail.

Here we report a detailed investigation of grain boundary precipitation in heat-treated and/or cold-rolled alloy 690 by coupling atom probe tomography (APT) with scanning and transmission electron microscopy, including electron backscatter diffraction techniques. The morphology of the carbides was significantly different between the alloys that were only heat-treated and the alloys that were cold-rolled prior to heat-treatment [2]. APT has revealed unprecedented detail about the local chemistry of the grain boundary carbides as well as other secondary precipitates.

Transmission electron microscopy (TEM) analysis identified that there are two different types of GB precipitates, fcc-based $\mathrm{Cr}_{23} \mathrm{C}_{6}$-type carbides and bcc alpha-Cr. The cold rolled alloy also exhibited a discontinuous Cr-lean phase in the vicinity of the grain-boundary after aging. APT studies also revealed Al-rich clusters near the carbides, possible an early stage of an as yet unknown phase that has formed inside the Cr-lean discontinuous product phase. Figure 1 shows the orientation maps of alloy 690 samples aged at $550{ }^{\circ} \mathrm{C}$ for $10000 \mathrm{~h}$ with and without cold rolling (CR). A significant influence of CR was observed on the grain boundary (GB) precipitation in this alloy, both carbide and alpha-Cr volume fraction increased due to $\mathrm{CR}$.

Raw ion maps from APT identified a carbide captured within the analysis volume from the alloy 690 aged at $475^{\circ} \mathrm{C}$ for $10000 \mathrm{~h}$. Though the bulk concentration of $\mathrm{Cr}$ is $33 \%$, local depletion of $\mathrm{Cr}$ (as low as $18 \%$ ) was noticed APT studies also revealed Al-rich clusters near the carbides, possible an early stage of an as yet unknown phase that has formed inside the Cr-lean discontinuous product phase (figure $2 \mathrm{f}$ ). A systematic and detailed analysis coupling Scanning electron microscopy (SEM), site specific lift out by using Focused Ion Beam (FIB), TEM and APT will be presented.

References:

[1] Was, G. S., Corrosion 46.4 (1990): 319-330.

[2] Thomas, Larry E., and Stephen M. Bruemmer.,Corrosion 56.6 (2000): 572-587. 


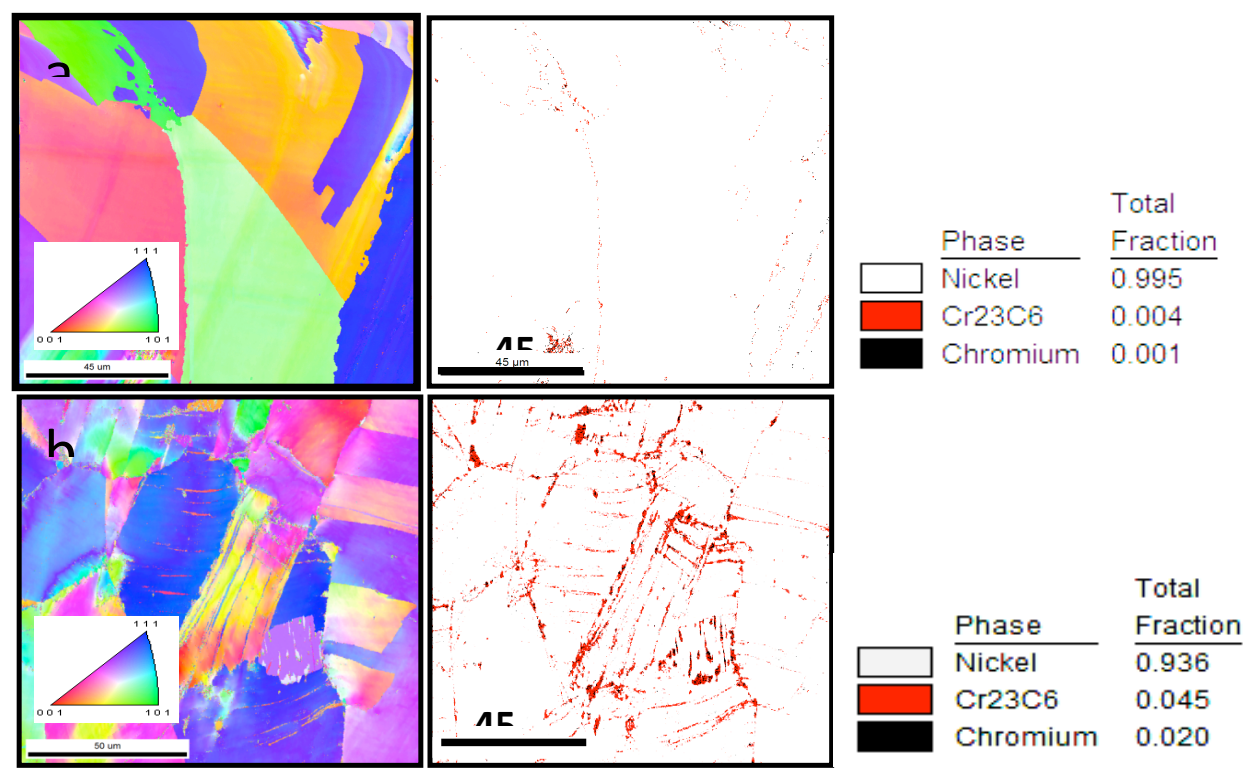

Figure 1. Orientation maps (accompanied by a unit triangle) by EBSD showing the orientation coloring scheme and phase fraction on their right(a) Annealed at $550{ }^{\circ} \mathrm{C}$ for $10000 \mathrm{~h}$ (b) $20 \%$ Cold rolled and annealed at $550{ }^{\circ} \mathrm{C}$ for $10000 \mathrm{~h}$
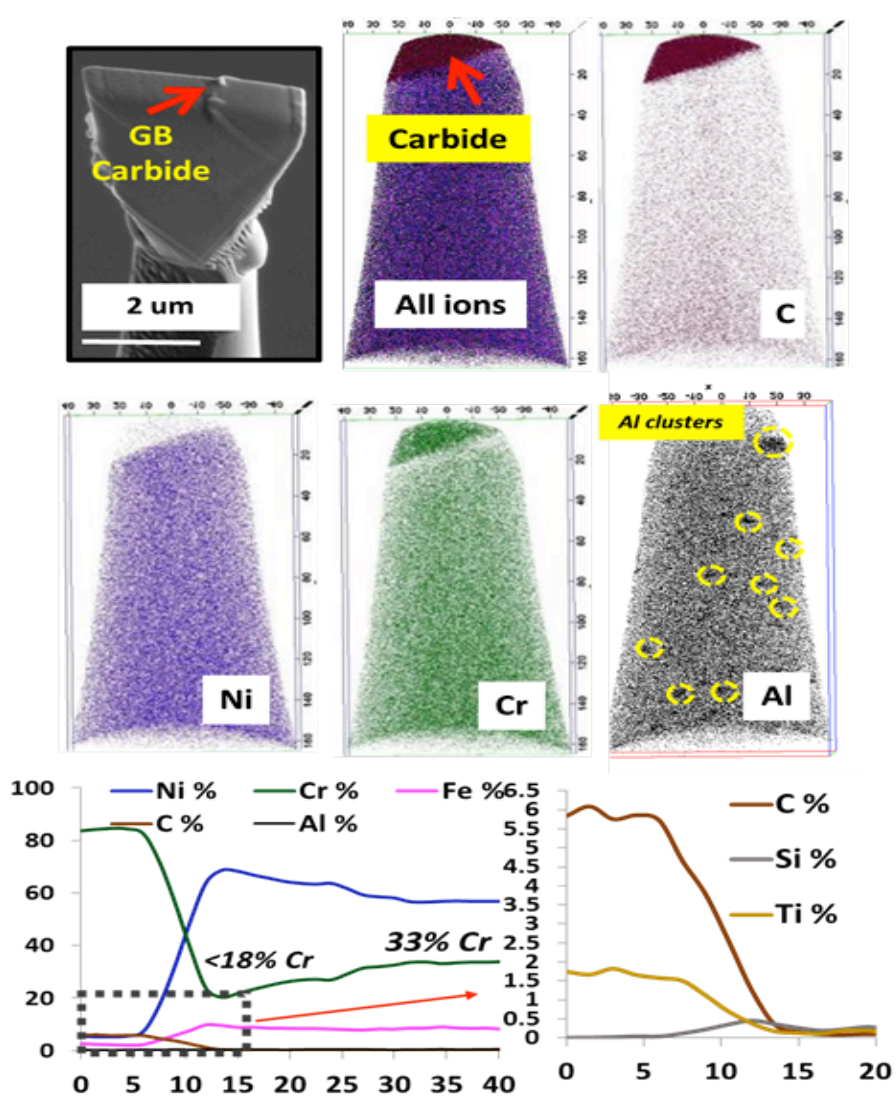

Figure 2. Grain boundary carbide analysis using APT on alloy 690 sample annealed at $475^{\circ} \mathrm{C}$ for $10000 \mathrm{~h}$ (a) Site of FIB lift out (b)-(f) ion maps showing marked ions (g) Proximity histogram showing the partitioning of major elements across a carbide-matrix interface $(\mathrm{h})$ partitioning of minor elements. Proximity histogram was generated by $60 \% \mathrm{Cr}$. 